\title{
Eficiência do hidrogel e respostas fisiológicas de mudas de cultivares apirênicas de citros sob défice hídrico ${ }^{1}$
}

\author{
Ester Alice Ferreira ${ }^{2}$, Vania Aparecida Silva ${ }^{2}$, \\ Elisângela Aparecida Silva ${ }^{3}$, Helbert de Rezende Oliveira Silveira ${ }^{4}$
}

\begin{abstract}
Hydrogel efficiency and physiological responses of seedless citrus cultivars seedlings under water deficit

Water is a limiting factor in citrus development which makes hydric replacement a common practice in plantations where its distribution is scarce. The hydroretentor gel has been one of the available technologies for water supply to plants and may also be an alternative that contributes to the rational use of water for planting citrus seedlings. This study evaluated the efficiency of hydrogel as an alternative to minimize the effects of water deficit in seedlings of seedless cultivars of tangerines ('Ortanique', 'Okitsu' and 'Clemenules') and oranges ('Navelina', 'Navelate' and 'Lanelate'), all grafted on Poncirus trifoliata. The experiment was carried out in a greenhouse, in a randomized blocks design, where plants with hydrogel were compared to plants under conventional irrigation and also to plants under water deficit, in a triple factorial arrangement. The rates of carbon liquid assimilation, stomatal conductance and transpiration and the ratio between internal and external $\mathrm{CO}_{2}$ concentrations were evaluated. It was verified that the effect of the hydrogel for maintaining the hydric status of citrus seedlings is variable and dependent on physiological mechanisms of response to water deficit. There was no response of 'Ortanique' and 'Navelate' seedlings to the hydrogel application. The hydrogel promoted the recovering and maintenance of the hydric status of 'Okitsu', 'Clemenules', 'Navelina' and 'Lanelate' seedlings, however, these cultivars were sensitive to changes in the water status, with considerable reduction of gas exchange.
\end{abstract}

KEY-WORDS: Citrus sp.; Poncirus trifoliata (L.) Raf.; soil conditioner; gas exchange.

\section{INTRODUÇÃO}

A citricultura é um dos segmentos mais fortes do agronégocio mundial e nacional, no qual são movimentados bilhões de dólares, a cada ano. Uma das principais tendências do mercado consumidor

\section{RESUMO}

A água é um dos fatores limitantes ao desenvolvimento de plantas cítricas, o que faz da reposição hídrica uma prática comum, em plantios onde a sua distribuição é escassa. O gel hidroretentor tem sido uma das tecnologias disponíveis de fornecimento de água para plantas, podendo ser uma alternativa no plantio de mudas cítricas, contribuindo para o uso racional da água. Neste trabalho, foi avaliada a eficiência do hidrogel como alternativa para minimizar os efeitos do défice hídrico, em mudas de cultivares apirênicas de tangerineiras ('Ortanique', 'Okitsu' e 'Clemenules') e de laranjeiras ('Navelina', 'Navelate' e 'Lanelate'), todas enxertadas sobre Poncirus trifoliata. $\mathrm{O}$ experimento foi conduzido em casa-de-vegetação, em delineamento de blocos casualizados, no qual plantas com hidrogel foram comparadas a plantas sob irrigação convencional e a plantas submetidas a estresse hídrico, em fatorial triplo. Foram determinadas as taxas de assimilação líquida de carbono, condutância estomática, transpiração e razão entre concentrações interna e externa de $\mathrm{CO}_{2}$. Verificou-se que o efeito do hidrogel na manutenção do status hídrico de mudas cítricas é variável e dependente de mecanismos fisiológicos de resposta ao défice hídrico. As mudas de 'Ortanique' e 'Navelate' não responderam à aplicação de hidrogel. Este promoveu recuperação e manutenção do status hídrico das mudas de 'Okitsu', 'Clemenules', 'Navelina' e 'Lanelate', porém, estas cultivares foram sensíveis a variações no status hídrico, apresentando redução considerável nas trocas gasosas.

PALAVRAS-CHAVE: Citrus sp.; Poncirus trifoliata (L.) Raf.; condicionador de solo; trocas gasosas.

deste setor, nos últimos anos, é o consumo de frutos apirênicos e, diante desta demanda, cultivares de laranja e tangerina sem sementes, já consagradas no mercado americano e europeu, foram introduzidas e avaliadas com sucesso, em algumas regiões do Brasil. Algumas delas se destacaram pelo melhor

1. Trabalho recebido em jul./2013 e aceito para publicação em jun./2014 (nº registro: PAT 25422).

2. Empresa de Pesquisa Agropecuária de Minas Gerais (Epamig), Unidade Regional Sul de Minas, Lavras, MG, Brasil.

E-mails: ester@epamig.br, vania.silva@epamig.br.

3. Universidade Federal de Lavras (UFLa), Departamento de Agricultura, Lavras, MG, Brasil. E-mail: agroelis@yahoo.com.br.

4. Universidade Federal de Lavras (UFLa), Departamento de Fisiologia Vegetal, Lavras, MG, Brasil.

E-mail: helbert_rezende@yahoo.com.br. 
desenvolvimento, como as laranjeiras 'Lanelate', 'Navelate'e 'Navelina' (Citrus sinensis (L.) Osbeck), as tangerineiras 'Clemenules' ( $C$. reticulata Blanco) e satsuma 'Okitsu' (C. unshiu Marcovitch) e o híbrido 'Ortanique' (tangor natural entre $C$. sinensis (L.) Osbeck e C. reticulata Blanco).

Além do diferencial da apirenia e da grande aceitação no mercado internacional, essas cultivares podem ser produzidas em diferentes épocas do ano, possibilitando a comercialização nas janelas de mercado (Oliveira et al. 2005, Pio 2003).

No Brasil, a exploração de citros é desenvolvida, em sua grande maioria, sob condições de sequeiro e, por isto, as plantas, frequentemente, são expostas às variações do clima, caracterizadas por episódios de seca ou distribuição irregular de chuvas, associadas a extremos de temperatura. Tais condições, comumente, causam défice hídrico nas plantas, afetando, negativamente, múltiplos parâmetros fisiológicos que determinam o crescimento e desenvolvimento das plantas de citros (Arbona et al. 2005). Neste contexto, o desenvolvimento de tecnologias que auxiliem as plantas a tolerar períodos prolongados de estiagem, bem como a utilização de cultivares mais tolerantes a períodos de défice hídrico, são essenciais para minimizar tais impactos negativos do défice hídrico.

Dentre as tecnologias disponíveis para o fornecimento de água às plantas, destacam-se os condicionadores de solo, também conhecidos como polímeros hidroabsorventes e géis hidroretentores e, ainda, como hidrogel, que, embora seja um termo genérico e usado em diversas áreas, tem sido amplamente utilizado na agricultura.

O hidrogel tem como principais características a capacidade de absorver 150 a 400 vezes a sua massa seca em água, habilidade de armazená-la e disponibilizá-la à planta, quando necessário, e, ainda, agir como agente tamponante contra o estresse hídrico temporário, minimizando os problemas associados à disponibilidade irregular ou deficitária de água (Azevedo et al. 2002, Prevedello \& Loyola 2007, Abedi-Koupai et al. 2008).

Essa tecnologia foi testada na manutenção do status hídrico de diversas espécies, como o Eucalyptus urophylla (Souza et al. 2006), Eucalyptus urograndis (Saad et al. 2009, Lopes et al. 2010), Casuarina glauca Sieber (El Hady et al. 2008) e outras nove espécies arbóreas (Orikiriza et al. 2009), bem como em diferentes genótipos de Brassica napus (Tohidi-Moghadam et al. 2009), Cucumis melon
(Demartelaere et al. 2009), Coffea canephora pierre (Zonta et al. 2009) e Coffea arabica L. (Marques et al. 2013), e, ainda, na sobrevivência de Jatropha curcas (Dranski et al. 2013), apresentando resultados variáveis.

A resposta das plantas ao estresse hídrico depende de sua intensidade e duração e é variável em cada espécie e fase de desenvolvimento, sendo, também, fortemente influenciada pela condição ambiental dos locais de estudo (Taiz \& Zeiger 2009), o que evidencia a necessidade de se estudarem os efeitos do hidrogel em diferentes espécies e situações.

Não se tem conhecimento sobre as respostas de cultivares de laranjas e tangerinas sem sementes ao défice hídrico e em cultivo com hidrogel. Para suprir esta lacuna e, considerando que, na fase de implantação do pomar, as mudas são mais sensíveis a défices de água, este estudo objetivou avaliar as respostas fisiológicas sob défice hídrico de mudas de cultivares apirênicas de citros e sua relação com a eficiência do hidrogel, na manutenção do status hídrico.

\section{MATERIAL E MÉTODOS}

A pesquisa foi desenvolvida em novembro de 2011, em casa-de-vegetação, sem controle de umidade e temperatura, na Empresa de Pesquisa Agropecuária de Minas Gerais (Epamig), Unidade Regional Sul de Minas, em Lavras (MG).

Foram instalados dois experimentos, nos quais foram avaliadas mudas das cultivares de tangerineiras 'Clemenules' (C. reticulata) e satsuma 'Okitsu' (C. unshiu) e do híbrido 'Ortanique' (C. sinensis $\mathrm{x}$ C. reticulata), no Experimento 1, e as laranjeiras 'Lanelate', 'Navelate' e 'Navelina' (C. sinensis), no Experimento 2.

Foram selecionadas mudas uniformes, com 18 meses de idade, as quais foram enxertadas sobre o porta-enxerto Poncirus Trifoliata. Para certificação da uniformidade das mudas, estas foram avaliadas e não apresentaram diferenças quanto à altura e diâmetro, com valores médios, respectivos, de $12,53 \mathrm{~cm}$ e $0,67 \mathrm{~cm}$, para tangerineiras, e de $13,25 \mathrm{~cm}$ e $0,65 \mathrm{~cm}$, para laranjeiras.

As mudas foram transplantadas para vasos de $22 \mathrm{~L}$ contendo substrato composto de terra de subsolo e areia, na proporção de 3:1. O solo foi analisado e apresentou $27 \%$ de argila, $53 \%$ de areia e $20 \%$ de silte, bem como as seguintes características: $\mathrm{pH}\left(\mathrm{H}_{2} \mathrm{O}\right)=5,8 ; \mathrm{P}=1,2 \mathrm{mg} \mathrm{dm}^{-3} ; \mathrm{K}=45 \mathrm{mg} \mathrm{dm}^{-3}$; 
$\left.\mathrm{Ca}^{+2}=1,3 \mathrm{cmol} \mathrm{dm}^{-3}\right) ; \mathrm{Mg}^{+2}=0,4 \mathrm{cmol} \mathrm{dm}^{-3}$; $\mathrm{Al}^{+3}=0,1 \mathrm{cmol} \mathrm{dm}^{-3} ; \mathrm{H}+\mathrm{Al}=2,3 \mathrm{cmol} \mathrm{dm}^{-3} ; \mathrm{SB}=$ $1,8 \mathrm{cmol} \mathrm{dm}^{-3} ; \mathrm{t}=1,9 \mathrm{cmol} \mathrm{dm}^{-3} ; \mathrm{T}=4,1 \mathrm{cmol} \mathrm{dm}^{-3}$; $\mathrm{V}=44 \% ; \mathrm{MO}=1,5 \mathrm{dag} \mathrm{kg}^{-1} ; \mathrm{P}-\mathrm{rem}=16 \mathrm{mg} \mathrm{L}^{-1}$.

Uma vez que os níveis de nutrientes foram satisfatórios, foi realizada uma adubação básica (Mattos Júnior et al. 2009) e, para determinar a quantidade de água a ser aplicada, determinou-se, também, a capacidade de campo do solo (Reichardt 1988).

Em cada experimento, as três cultivares foram avaliadas em três sistemas de cultivo: 1) convencional (com irrigação periódica, mantendo-se o solo próximo à sua capacidade de campo);2) com défice hídrico (plantas submetidas a deficiência hídrica, por meio de suspensão total da rega); 3) com hidrogel.

As características do hidrogel utilizado estão descritas em Hydroplan-EB (2001) e este foi preparado seguindo-se recomendação do fabricante, utilizando-se a formulação de $4 \mathrm{~g}$ do produto para cada litro de água. Cada vaso recebeu $2 \mathrm{~L}$ do gel expandido, colocados na base dos mesmos, seguidos pela muda a ser avaliada.

Utilizou-se o delineamento em blocos casualizados, com duas plantas por parcela e cinco repetições, constituindo um fatorial triplo $3 \times 3 \times 2$ (cultivar x sistema de cultivo x época de avaliação). As avaliações foram realizadas em duas épocas: no primeiro e no décimo quinto dia após a imposição do défice hídrico, ocasião em que foi atingido o défice moderado, considerado limite para avaliação das trocas gasosas.

As respostas fisiológicas foram mensuradas pelo potencial hídrico na antemanhã $\left(\psi_{\mathrm{am}}\right)$ e ao meio-dia $\left(\psi_{\text {md }}\right)$, com o auxílio de uma bomba de pressão tipo Scholander, e pelas trocas gasosas quantificadas entre $07 \mathrm{~h} 00$ e $08 \mathrm{~h} 30$. As taxas de assimilação líquida de carbono $(A)$, condutância estomática $\left(g_{s}\right)$, transpiração $(E)$ e razão entre concentrações interna e externa de $\mathrm{CO}_{2}\left(C_{\mathrm{i}} / C_{\mathrm{a}}\right)$ foram medidas em sistema aberto, sob luz saturante artificial $\left(1.200 \mathrm{mmol} \mathrm{m}^{-2} \mathrm{~s}^{-1}\right)$ e concentração de $\mathrm{CO}_{2}$ ambiente, sob temperatura constante de $25^{\circ} \mathrm{C}$, com o auxílio de analisador de gás a infravermelho (IRGA) portátil (LICOR 6400, LiCOR, Nebraska, USA).

Para a análise estatística, verificou-se, inicialmente, se os dados das variáveis analisadas atendiam às pressuposições básicas de normalidade e homocedasticidade, no programa R (RFSC 2011), e, sendo atendidos estes parâmetros, procedeu-se à análise de variância e às médias dos tratamentos, que foram comparadas entre si pelo teste de Skott-Knott, a 5\%, pelo Sisvar (Ferreira 2011).

\section{RESULTADOS E DISCUSSÃO}

Os valores médios de temperatura, radiação fotossinteticamente ativa e défice de pressão de vapor registrados pelo analisador infravermelho, nas tangerineiras avaliadas no Experimento 1, foram, respectivamente: $31,36^{\circ} \mathrm{C}$ e $32,45^{\circ} \mathrm{C} ; 584,45 \mu \mathrm{mol} \mathrm{m} \mathrm{m}^{-2} \mathrm{~s}^{-1}$ e $378,66 \mu \mathrm{mol} \mathrm{m}{ }^{-2} \mathrm{~s}^{-1}$; e $3,03 \mathrm{kPa}$ e $2,59 \mathrm{kPa}$, no primeiro e no décimo quinto dia de suspensão de rega. Estas variações ambientais entre as datas de avaliação não influenciaram, significativamente, nas trocas gasosas das cultivares irrigadas, com exceção dos valores de condutância estomática da cultivar 'Ortanique', que foram maiores em condições de menor temperatura, e demais variáveis. Ressalta-se, portanto, que as variações observadas entre os tratamentos, nas diferentes épocas, devem-se ao efeito do hidrogel e do défice hídrico.

No primeiro dia da suspensão da rega, verificou-se que as diferentes cultivares de tangerineiras não apresentaram diferenças, quanto aos potenciais hídricos na antemanhã $\left(\psi_{\mathrm{am}}\right)$ e ao meio-dia $\left(\psi_{\mathrm{md}}\right)$, quando conduzidas em substrato com e sem a adição de hidrogel (Tabela 1). A aplicação de hidrogel não alterou, também, as trocas gasosas, em condições de disponibilidade hídrica. Comparando-se as cultivares, observou-se que, de maneira geral, a condutância estomática da 'Ortanique' apresentou-se, em média, 38\% e 53\% superior à das cultivares 'Okitsu' e 'Clemenules', na primeira e segunda avaliação, respectivamente. Entretanto, a assimilação líquida de $\mathrm{CO}_{2}$, transpiração e razão $C_{\mathrm{i}} / C_{\mathrm{a}}$ não diferiram entre as cultivares (Tabela 1).

Após 15 dias da suspensão da irrigação, a 'Ortanique' não apresentou redução significativa nos potenciais hídricos na antemanhã $\left(\psi_{\mathrm{am}}\right)$ e ao meio-dia $\left(\psi_{\mathrm{md}}\right)$ e, portanto, manteve a taxa fotossintética, condutância estomática e transpiração semelhantes às observadas para as plantas irrigadas. Já as cultivares 'Okitsu' e 'Clemenules' submetidas ao défice hídrico apresentaram redução de ambos os potenciais, ressaltando-se que a 'Clemenules' apresentou os menores potenciais hídricos. O défice hídrico, nestas duas cultivares, causou decréscimos, também, nos valores dos parâmetros de trocas gasosas, sendo que, na cultivar 'Okitsu', houve reduções de 54\% em $A$ e de $53 \%$ em $g_{\text {s }}$, acompanhadas por diminuição 


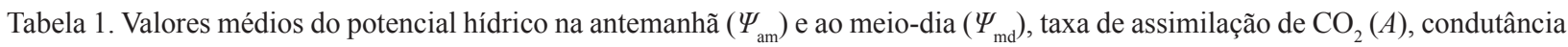
estomática $\left(g_{\mathrm{s}}\right)$, transpiração $(E)$ e razão entre concentrações interna e externa de $\mathrm{CO}_{2}\left(C_{\mathrm{i}} / C_{\mathrm{a}}\right)$, em cultivares apirênicas de tangerineiras submetidas a diferentes tratamentos: irrigação convencional (IC), com hidrogel (CH) e estresse hídrico (EH) (Lavras, MG, 2011).

\begin{tabular}{|c|c|c|c|c|c|c|c|}
\hline \multirow{2}{*}{\multicolumn{2}{|c|}{ Parâmetro }} & \multicolumn{3}{|c|}{ Primeiro dia de suspensão da rega } & \multicolumn{3}{|c|}{ Décimo quinto dia de suspensão da rega } \\
\hline & & $\mathrm{IC}$ & $\mathrm{CH}$ & $\mathrm{EH}$ & $\mathrm{IC}$ & $\mathrm{CH}$ & EH \\
\hline \multirow{3}{*}{$\Psi_{\mathrm{am}}(\mathrm{MPa})$} & 'Ortanique' & 2,25 Aaa* & 2,87 Aaa & 2,87 Aaa & 3,00 Aạa & 4,87 Bạa & $5,50 \mathrm{Ca} a$ \\
\hline & 'Okitsu’ & 2,50 Aạa & $2,62 \mathrm{~A} \underline{\mathrm{b}} \mathrm{a}$ & $2,50 \mathrm{~A} \underline{\mathrm{b}} \mathrm{a}$ & $2,87 \mathrm{Aac}$ & $8,50 \mathrm{Aab}$ & $13,62 \mathrm{Baa}$ \\
\hline & 'Clemenules' & 3,12 Aa a & $3,00 \mathrm{Ab}$ & $3,25 \mathrm{Ab} a$ & $3,25 \mathrm{Aac}$ & $11,37 \mathrm{Aab}$ & $25,50 \mathrm{Aa} a$ \\
\hline \multirow{3}{*}{$\Psi_{\mathrm{md}}(\mathrm{MPa})$} & 'Ortanique' & 12,37 Aạa & 13,00 Aạa & 14,75 Aạa & 10,00 Aạa & $14,37 \mathrm{Baa}$ & $13,00 \mathrm{Ca} a$ \\
\hline & 'Okitsu’' & 17,00 Aạa & $16,00 \mathrm{~A} \underline{\mathrm{b}} \mathrm{a}$ & 16,12 Aạa & 12,50 Aạa & $22,50 \mathrm{Aa} b$ & $21,87 \mathrm{Ba} b$ \\
\hline & 'Clemenules' & 13,87 Aạa & $15,12 \mathrm{Ab}$ & $15,25 \mathrm{Ab} a$ & $10,12 \mathrm{Aa} a$ & $25,25 \mathrm{Aa} b$ & $32,50 \mathrm{Aac}$ \\
\hline \multirow{3}{*}{$A\left(\mu \mathrm{mol} \mathrm{m} \mathrm{m}^{-2} \mathrm{~s}^{-1}\right)$} & 'Ortanique' & 8,61 Aạa & 8,98 Aạa & $8,15 \mathrm{Aaa}$ & 9,04 Aạa & 8,04 Aạa & 6,46 Aạa \\
\hline & 'Okitsu’ & 7,88 Aạa & 8,48 Aạa & 6,67 Aạa & 8,56 Aạa & $3,84 \mathrm{~B} \underline{b} b$ & $3,98 \mathrm{Bab}$ \\
\hline & 'Clemenules' & 7,81 Aaa & 8,08 Aaa & 7,83 Aaa & 7,53 Aaa & $2,49 \mathrm{~B} \underline{b} b$ & $1,38 \mathrm{~B} \underline{b} b$ \\
\hline \multirow{3}{*}{$g_{\mathrm{s}}\left(\mathrm{mmolm}^{-2} \mathrm{~s}^{-1}\right)$} & 'Ortanique' & $0,15 \mathrm{Aaa} a$ & $0,17 \mathrm{Aaa} a$ & 0,17 Aạa & $0,25 \mathrm{Ab} a$ & $0,20 \mathrm{Aa} a \mathrm{a}$ & 0,18 Aạa \\
\hline & 'Okitsu' & 0,12 Aaa & 0,10 Aạa & $0,085 \mathrm{Baaa}$ & 0,17 Bạa & $0,067 \mathrm{~B} \underline{a} b$ & 0,08 Bạb \\
\hline & 'Clemenules' & 0,09 A $\underline{a} a$ & 0,09 A $\underline{a} \mathrm{a}$ & $0,097 \mathrm{Ba} a$ & $0,167 \mathrm{~B} \underline{\mathrm{aa}}$ & $0,037 \mathrm{Bab}$ & $0,027 \overline{\mathrm{B}} \underline{\mathrm{ab}}$ \\
\hline \multirow{3}{*}{$E\left(\mu \mathrm{mol} \mathrm{m} \mathrm{m}^{-2} \mathrm{~s}^{-1}\right)$} & 'Ortanique' & 4,05 Aạa & 4,26 Aaa & 4,22 Aạa & 4,97 Aaa & 3,92 Aạa & 4,17 Aaa \\
\hline & 'Okitsu' & 3,36 Aāa & $3,17 \mathrm{Aa} a$ & 2,44 Bạa & $4,07 \mathrm{Aa} a$ & $1,85 \mathrm{Ba} b$ & $2,21 \mathrm{Ba} b$ \\
\hline & 'Clemenules' & 2,64 Aaa & 2,84 Aạa & $2,84 \mathrm{Baa}$ & $3,75 \mathrm{Aa} a$ & $1,13 \mathrm{~B} \underline{b} b$ & $0,80 \mathrm{~B} \underline{b} b$ \\
\hline \multirow{3}{*}{$C_{\mathrm{i}} / C_{\mathrm{a}}$} & 'Ortanique' & 0,72 Aạa & $0,72 \mathrm{Aa} a$ & $0,74 \mathrm{Aa} a \mathrm{a}$ & $0,81 \mathrm{Aa} a \mathrm{a}$ & $0,81 \mathrm{Aa} a \mathrm{a}$ & $0,80 \mathrm{Aa} a \mathrm{a}$ \\
\hline & 'Okitsu’' & $0,61 \mathrm{Aa} a$ & $0,67 \mathrm{Aa} a$ & $0,73 \mathrm{Aa} a$ & $0,75 \mathrm{Aa} a \mathrm{a}$ & 0,71 Aạa & 0,74 Aạa \\
\hline & 'Clemenules' & $0,62 \mathrm{Aa} a$ & $0,60 \mathrm{Aa} a$ & $0,64 \mathrm{Aa} a$ & $0,68 \mathrm{Aa} a$ & $0,65 \mathrm{Aa} a$ & $0,74 \mathrm{Aa} a \mathrm{a}$ \\
\hline
\end{tabular}

* Letras maiúsculas, na coluna, comparam diferentes cultivares no mesmo tratamento. Letras minúsculas sublinhadas, na mesma linha, comparam o mesmo tratamento (hidrogel), nas diferentes épocas. Letras minúsculas, na mesma linha, comparam diferentes tratamentos (hidrogel), na mesma época.

de $46 \%$ em $E$, em relação às plantas com irrigação convencional. Para a cultivar 'Clemenules', as reduções de $A, g_{s}$ e $E$ foram de, aproximadamente, $82 \%$, $84 \%$ e $79 \%$, respectivamente. Estas diminuições não foram acompanhadas por menores valores de $C_{\mathrm{i}} / C_{\mathrm{a}}$, sugerindo que a menor $A$ pode ser atribuída tanto a limitações estomáticas, pela diminuição da disponibilidade de $\mathrm{CO}_{2}$ no mesofilo e sítios de carboxilação, como não estomáticas, determinadas, possivelmente, pela inativação parcial de rubisco, bem como pela quantidade desta enzima (Ribeiro \& Machado 2007).

Quanto à efetividade do hidrogel na manutenção do status hídrico das cultivares, após 15 dias da suspensão da irrigação, observou-se que, para 'Ortanique', o $\psi_{\text {am }}$ e o $\psi_{\text {md }}$ das plantas cultivadas com hidrogel não diferiram das plantas irrigadas e das plantas submetidas ao défice hídrico, e que esta cultivar manteve o potencial hídrico, independentemente da adição de hidrogel ao substrato.

Essa manutenção do status hídrico não está relacionada à desuniformidade das mudas e, uma vez que a cultivar 'Ortanique' manteve maior $g_{\mathrm{s}}$, após a suspensão da irrigação, o mecanismo de redução da condutância estomática para reduzir a transpiração e, consequentemente, a perda de água, também não ex- plicaria a manutenção do status hídrico. A capacidade de esta cultivar manter potenciais hídricos foliares adequados pode ser atribuída a características bioquímicas relacionadas à manutenção da capacidade de transporte de fotoassimilados da parte aérea para as raízes, possibilitando a absorção de água (Rewald et al. 2012).

Embora as cultivares estejam sobre o mesmo porta-enxerto (Poncirus trifoliata), a combinação de diferentes copas pode influenciar no desenvolvimento do sistema radicular, por meio de mudanças nos mecanismos fisiológicos da planta, como o balanço hormonal, absorção de água, trocas gasosas e translocação de assimilados (Jover et al. 2012).

Para a 'Okitsu', o $\psi_{\text {am }}$ das plantas cultivadas com hidrogel foi menor, em relação às plantas mantidas irrigadas, porém, maior do que o das plantas submetidas ao défice hídrico sem adição de hidrogel, no entanto, observou-se que a utilização do gel no substrato permitiu uma recuperação do status hídrico das mudas desta cultivar. $\mathrm{O} \psi_{\text {am }}$ indica a condição hídrica da planta, podendo refletir o potencial de água na região da rizosfera, uma vez que planta e rizosfera permanecem em equilíbrio, durante a noite (Kavanagh et al. 2007). 
Paralelamente ao $\psi_{\text {am }}$, os menores valores médios de potencial hídrico registrados ao meio-dia $\left(\psi_{\mathrm{md}}\right)$, nas mudas de 'Okitsu' cultivadas com gel, foram semelhantes aos das plantas sob défice hídrico, refletindo a maior demanda atmosférica de vapor d'água neste período do dia, quando comparado ao período da antemanhã. Além disto, este comportamento pode ser um indicativo de baixa condutividade hidráulica e/ou maior rigidez da parede celular desta cultivar, que se traduzem em rápido decréscimo do potencial de parede, para uma perda de água relativamente pequena, resultando em rápido declínio de $\psi_{\text {md }}$ (Damatta \& Rena 2001).

Para a cultivar 'Clemenules', ambos os potenciais hídricos $\left(\psi_{\mathrm{am}} \mathrm{e} \psi_{\mathrm{md}}\right)$ das plantas cultivadas com hidrogel foram menores, em relação às plantas irrigadas, porém, superiores aos das plantas submetidas ao défice hídrico sem adição de hidrogel. Isto permite inferir que o hidrogel foi mais eficiente em manter o potencial hídrico na cultivar mais sensível, ou seja, na que atinge potenciais hídricos mais severos em condições de défice hídrico.

Embora a utilização do gel no substrato tenha permitido recuperação e manutenção parcial do status hídrico das mudas de 'Okitsu' e 'Clemenules', isto não foi suficiente para manter as trocas gasosas iguais às das plantas irrigadas, pois houve redução de $A, g_{\mathrm{s}}$ e $E$, conforme ocorreu nas mudas submetidas ao défice, em substrato sem gel. Estes resultados permitem inferir que as cultivares 'Clemenules' e 'Okitsu' são bastante sensíveis a variações do status hídrico, uma vez que os défices hídricos das mudas cultivadas em gel já foram suficientes para causar efeitos negativos expressivos, nas trocas gasosas destas cultivares. Desta maneira, em cultivares sensíveis a variações do status hídrico, o hidroretentor pode não ter efeito sobre o crescimento de mudas, conforme já foi verificado em outras culturas, como o café (Vale et al. 2006).

Considerando-se o Experimento 2, com laranjeira, os valores médios de temperatura, radiação fotossinteticamente ativa e défice de pressão de vapor, no primeiro e no décimo quinto dia de suspensão de rega, foram, respectivamente: $34,89^{\circ} \mathrm{C}$ e $33,16^{\circ} \mathrm{C} ; 606,54 \mathrm{mmol} \mathrm{m}^{-2} \mathrm{~s}^{-1}$ e $264,82 \mathrm{mmol} \mathrm{m}^{-2} \mathrm{~s}^{-1}$; e 2,43 $\mathrm{kPa}$ e 2,31 kPa. Apesar destas variações ambientais, não houve diferenças entre as trocas gasosas das cultivares irrigadas, nas duas datas avaliadas, $o$ que evidencia que as variações observadas entre os tratamentos, nas diferentes épocas, deve-se ao efeito do hidrogel e do défice hídrico.
No Experimento 2, houve resposta de diferentes cultivares de laranjeira à aplicação de hidrogel, em relação ao potencial hídrico na antemanhã $\left(\psi_{\mathrm{am}}\right)$ e ao meio-dia $\left(\psi_{\text {md }}\right)$. Verificou-se que, no dia da suspensão da rega, não houve diferenças entre as cultivares de laranjeira mantidas com e sem adição de hidrogel e sob irrigação (Tabela 2). Nestas condições, as taxas de fotossíntese líquida $(A)$, condutância estomática $\left(g_{\mathrm{s}}\right)$, transpiração $(E)$ e razão $C_{\mathrm{i}} / C_{\mathrm{a}}$ não foram diferentes entre as mudas irrigadas e contendo ou não hidrogel. Estes resultados indicam que a presença de hidrogel não altera as trocas gasosas das mudas destas cultivares, corroboraando os resultados obtidos por Arbona et al. (2005).

Após 15 dias da suspensão da irrigação, todas as cultivares submetidas ao défice hídrico apresentaram redução do potencial hídrico na antemanhã $\left(\psi_{\mathrm{am}}\right)$ e ao meio-dia $\left(\psi_{\mathrm{md}}\right)$, quando comparadas às plantas irrigadas.

O défice hídrico causou decréscimos, também, nos valores dos parâmetros de trocas gasosas das cultivares conduzidas em substrato sem a adição do hidroretentor. De maneira geral, houve reduções médias de $55 \%$ em $A$ e de $78 \%$ em $g_{\text {s }}$, acompanhadas por diminuição de $64 \%$ em $E$, quando comparadas às cultivares irrigadas. Quanto ao parâmetro $C_{\mathrm{i}} / C_{\mathrm{a}}$, houve redução de $25 \%$ sob défice hídrico, para as cultivares 'Navelina' e 'Navelate'. O decréscimo em $A$, acompanhado pela redução em $g_{\mathrm{s}}$ e na razão $C_{\mathrm{i}} / C_{\mathrm{a}}$, sugere limitações estomáticas à fotossíntese, pela diminuição da disponibilidade de $\mathrm{CO}_{2}$ no mesofilo e sítios de carboxilação (Machado et al. 2006). Não ocorreu redução na razão $C_{\mathrm{i}} / C_{\mathrm{a}}$ para a cultivar 'Lanelate' sob défice hídrico, sugerindo que a inibição fotossintética possa ter ocasionado, também, uma inibição não estomática da fotossíntese, determinada, possivelmente, pela inativação parcial da enzima ribulose difosfato-carboxilase, bem como pela quantidade desta enzima (Ribeiro et al. 2009).

Os valores para trocas gasosas estão dentro da faixa de variação observada na literatura, para citros, conforme reportado por Perez-Perez et al. (2007). Segundo Ribeiro \& Machado (2007), plantas perenes com metabolismo $\mathrm{C} 3$, como as cítricas, mostram baixas taxas fotossintéticas, em condições naturais, com valores máximos de, aproximadamente, $13 \mu \mathrm{mol} \mathrm{m}{ }^{-2} \mathrm{~s}^{-1}$.

Não foram verificadas diferenças significativas entre as cultivares mantidas em substrato sem adição de hidrogel sob défice hídrico, evidenciando uma 


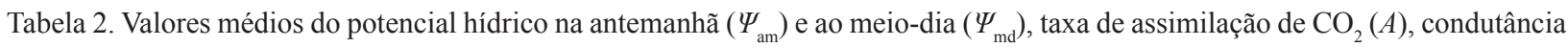
estomática $\left(g_{\mathrm{s}}\right)$, transpiração $(E)$ e razão entre concentrações interna e externa de $\mathrm{CO}_{2}\left(C_{\mathrm{i}} / C_{\mathrm{a}}\right)$, em cultivares apirênicas de laranjeiras submetidas a diferentes tratamentos: irrigação convencional (IC); com hidrogel $(\mathrm{CH})$ e estresse hídrico (EH) (Lavras, MG, 2011).

\begin{tabular}{|c|c|c|c|c|c|c|c|}
\hline \multirow{2}{*}{\multicolumn{2}{|c|}{ Parâmetro }} & \multicolumn{3}{|c|}{ Primeiro dia de suspensão da rega } & \multicolumn{3}{|c|}{ Décimo quinto dia de suspensão da rega } \\
\hline & & IC & $\mathrm{CH}$ & $\mathrm{EH}$ & $\mathrm{IC}$ & $\mathrm{CH}$ & $\mathrm{EH}$ \\
\hline \multirow{3}{*}{$\Psi_{\mathrm{am}}(\mathrm{MPa})$} & 'Navelina' & 2,12 Aạa & 2,75 Aạa & $2,12 \mathrm{~A} \underline{\mathrm{b}} \mathrm{a}$ & 2,50 Aac & $10,75 \mathrm{Ab} b \mathrm{~b}$ & 23,00 Aạa \\
\hline & 'Lanelate' & 2,62 Aạa & 2,75 Aạa & $2,37 \mathrm{~A} \underline{\mathrm{b}} \mathrm{a}$ & $2,25 \mathrm{Aac}$ & 9,12 Aa $\underline{a b}$ & 23,75 A ạa \\
\hline & 'Navelate' & 2,00 Aạa & $2,50 \mathrm{~A} \underline{\mathrm{b}} \mathrm{a}$ & $2,16 \mathrm{~A} \underline{b} \mathrm{a}$ & $2,25 \mathrm{~A} \underline{\mathrm{a}} \mathrm{b}$ & $25,00 \mathrm{Ba} a$ & 19,87 A $\underline{a} a$ \\
\hline \multirow{3}{*}{$\Psi_{\mathrm{md}}(\mathrm{MPa})$} & 'Navelina' & 11,62 Aạa & 8,50 Aạa & $8,87 \mathrm{~A} \underline{\mathrm{b} a}$ & $7,00 \mathrm{Aac}$ & $16,50 \mathrm{Aa} \underline{\mathrm{ab}}$ & 30,25 Aạa \\
\hline & 'Lanelate' & 10,25 A $\underline{a} a$ & 9,12 A $\underline{\text { aaa }}$ & $8,87 \mathrm{~A} \underline{\mathrm{b}} \mathrm{a}$ & $5,00 \mathrm{Aac}$ & $17,87 \mathrm{~A} \underline{\mathrm{a}} \mathrm{b}$ & 33,50 A $\underline{a} a$ \\
\hline & 'Navelate' & $10,50 \mathrm{Aa} a$ & $4,37 \mathrm{~A} \underline{\mathrm{b}} \mathrm{a}$ & $7,00 \mathrm{~A} \underline{\mathrm{b}} \mathrm{a}$ & $4,37 \mathrm{Aac}$ & $31,33 \mathrm{Baa}$ & $24,50 \mathrm{Aa}$ \\
\hline \multirow{3}{*}{$A\left(\mu \mathrm{mol} \mathrm{m} \mathrm{m}^{-2} \mathrm{~s}^{-1}\right)$} & 'Navelina' & 9,01 Aạa & 10,96 Aạa & 8,08 Aaaa & 9,96 Aaaa & $6,26 \mathrm{Ab} b \mathrm{~b}$ & $3,96 \mathrm{Ab} b \mathrm{~b}$ \\
\hline & 'Lanelate' & 8,83 Aạa & 9,11 Aạa & 10,29 Aạa & 9,72 Aaaa & 5,59 A $\underline{b} b$ & $3,16 \mathrm{~A} \underline{b} \mathrm{~b}$ \\
\hline & 'Navelate' & 8,39 Aaa & 9,37 Aaa & 10,14 Aaa & 9,63 Aaa & $1,78 \mathrm{~B} \underline{b} b$ & $4,01 \mathrm{~A} \underline{\mathrm{b}} \mathrm{b}$ \\
\hline \multirow{3}{*}{$g_{s}\left(\mathrm{mmol} \mathrm{m}{ }^{-2} \mathrm{~s}^{-1}\right)$} & 'Navelina' & 0,147 Aạa & 0,162 Aạa & 0,167 Aạa & 0,160 Aạa & 0,100 Aạa & $0,032 \mathrm{~A} \underline{b} b$ \\
\hline & 'Lanelate' & $0,100 \mathrm{~A} \underline{\mathrm{a} a}$ & 0,162 A $\underline{a} a$ & 0,167 Aaa & 0,175 A $\underline{a} a$ & $0,057 \mathrm{~A} \underline{\mathrm{b}} \mathrm{b}$ & $0,037 \mathrm{~A} \underline{b} b$ \\
\hline & 'Navelate' & $0,137 \mathrm{Aaa}$ & $0,132 \mathrm{~A} \underline{\mathrm{aa}}$ & $0,115 \mathrm{Aa} \underline{\mathrm{aa}}$ & $0,142 \mathrm{~A} \underline{\mathrm{a} a}$ & $0,022 \mathrm{Ab} \underline{b} \mathrm{~b}$ & $0,032 \mathrm{~A} \underline{\mathrm{b}} \mathrm{b}$ \\
\hline \multirow{3}{*}{$E\left(\mu \mathrm{mol} \mathrm{m} \mathrm{m}^{-2} \mathrm{~s}^{-1}\right)$} & 'Navelina' & 4,93 Aạa & 5,29 Aạa & 3,97 Aạa & 4,10 Aạa & $2,40 \mathrm{Ab} b \mathrm{~b}$ & $1,09 \mathrm{~A} \underline{b b}$ \\
\hline & 'Lanelate' & 3,38 Aaa & 5,08 Aạa & 5,40 Aạa & 4,24 Aaa & $1,78 \mathrm{~A} \underline{b} b$ & $1,25 \mathrm{~A} \underline{b} \mathrm{~b}$ \\
\hline & 'Navelate' & 4,69 Aaa & 4,83 Aaa & 5,34 Aaa & 3,91 Aaa & $0,71 \mathrm{~A} \underline{b} b$ & $2,04 \mathrm{~A} \underline{b} \mathrm{~b}$ \\
\hline \multirow{3}{*}{$C_{\mathrm{i}} / C_{\mathrm{a}}$} & 'Navelina' & 0,67 Aaa & 0,65 Aaa & 0,64 Aaa & 0,69 Aaa & $0,53 \mathrm{Aab}$ & $0,45 \mathrm{~A} \underline{b} \mathrm{~b}$ \\
\hline & 'Lanelate' & $0,59 \mathrm{Aa} a$ & $0,64 \mathrm{Aa} a$ & $0,67 \mathrm{Aa} a$ & 0,68 Aaa & $0,52 \mathrm{~A} \underline{b} \mathrm{~b}$ & $0,62 \mathrm{Baa}$ \\
\hline & 'Navelate' & $0,68 \mathrm{Aa} a$ & $0,64 \mathrm{Aa} a$ & 0,69 Aa a & 0,66 Aaа & $0,61 \mathrm{Aa} a$ & $0,46 \mathrm{~A} \underline{b} \mathrm{~b}$ \\
\hline
\end{tabular}

* Letras maiúsculas, na coluna, comparam diferentes cultivares, no mesmo tratamento. Letras minúsculas sublinhadas, na mesma linha, comparam o mesmo tratamento (hidrogel), nas diferentes épocas. Letras minúsculas, na mesma linha, comparam diferentes tratamentos (hidrogel), na mesma época.

semelhança entre a tolerância das cultivares, quanto ao estresse. Contudo, foram constatadas diferenças entre as respostas das cultivares ao défice hídrico, em substrato com hidrogel. Neste caso, 'Navelate' mostrou menores valores de $\psi_{\text {am }}$ e $\psi_{\text {md }}$ que as cultivares 'Navelina' e 'Lanelate'. A menor manutenção do status hídrico na 'Navelate' refletiu, também, em menores valores de assimilação líquida de carbono. A referida cultivar mostrou redução de $\psi_{\text {am }}$ semelhante à observada nas plantas conduzidas sob défice hídrico, o que evidencia ausência de efeito do hidrorretentor. Por outro lado, nas cultivares 'Navelina' e 'Lanelate', $\mathrm{o} \psi_{\text {am }} \mathrm{e} \psi_{\text {md }}$ foram maiores nas plantas cultivadas com hidrogel do que nas plantas cultivadas sem hidrogel, mostrando efeito positivo do hidrogel na manutenção do status hídrico destas cultivares, conforme verificado para cafeeiro (Zonta et al. 2009, Marques et al. 2013).

À semelhança das tangerineiras, a eficiência do hidrogel mostrou-se largamente dependente da resposta fisiológica da cultivar ao défice hídrico. Logo, o hidrogel não foi eficiente em manter o status hídrico da cultivar 'Navelate', que reduziu, pronunciadamente, o potencial hídrico e as trocas gasosas, na presença de hidrogel, comparativamente às outras cultivares estudadas. Uma vez que não houve diferenças significativas entre as cultivares mantidas em substrato sem adição de hidrogel, tanto irrigadas quanto sob défice hídrico, a sensibilidade, nesta cultivar, pode estar relacionada à menor eficiência hidráulica, densidade de venação ou à menor capacidade de absorção de água disponível no hidrogel pelas raízes (Brodribb et al. 2010).

Já para as cultivares 'Navelina' e 'Lanelate', o hidrogel permitiu maior manutenção do status hídrico das mudas. Contudo, esta manutenção não foi suficiente para manter as trocas gasosas iguais às das plantas irrigadas, pois houve redução de $A$, $E$ e $C_{\mathrm{i}} / C_{\mathrm{a}}$, conforme ocorreu nas mudas submetidas ao défice, em substrato sem gel. No entanto, a habilidade do hidrogel para reter e disponibilizar água mais lentamente pode favorecer a resiliência, ou seja, a habilidade de as mudas retornarem ao estado normal, após a ocorrência do estresse hídrico (Agaba et al. 2011).

\section{CONCLUSÕES}

1. As mudas de 'Ortanique' apresentaram manutenção do status hídrico e das trocas gasosas, independen- 
temente da aplicação de hidrogel, sendo, portanto, uma característica da cultivar.

2. As mudas de 'Navelate' reduziram, pronunciadamente, o potencial hídrico e as trocas gasosas, independentemente da aplicação de hidrogel.

3. O hidrogel promoveu recuperação e manutenção do status hídrico das mudas de 'Okitsu', 'Clemenules', 'Navelina' e 'Lanelate', porém, estas cultivares mostraram-se sensíveis a variações no status hídrico, apresentando redução considerável nas trocas gasosas.

\section{AGRADECIMENTOS}

À Fundação de Amparo à Pesquisa do Estado de Minas Gerais (Fapemig), pelo apoio financeiro, e à Hydroplan-EB, pelo fornecimento do hidrogel utilizado nos experimentos.

\section{REFERENNCIAS}

ABEDI-KOUPAI, J.; SOHRAB, F.; SWARBRICK, G. Evaluation of hydrogel application on soil water retention characteristics. Journal of Plant Nutrition, London, v. 31, n. 2, p. 317-331, 2008.

AGABA, H. et al. Hydrogel amendment to sandy soil reduces irrigation frequency and improves the biomass of Agrostis stolonifera. Agricultural Sciences, Coulterville, v. 2, n. 4, p. 544-550, 2011.

ARBONA, V. et al. Hydrogel substrate amendment alleviates drought effects on young citrus. Plant and Soil, Crawley, v. 270, n. 1, p. 73-82, 2005.

AZEVEDO, T. L. F.; BERTONHA, A.; GONCALVEZ, A. C. A. Uso de hidrogel na agricultura. Revista do Programa de Ciências Agroambientais, Alta Floresta, v. 1, n. 1, p. 23-31, 2002.

BRODRIBB, T. J.; FIELD, T. S.; SACK, L. Viewing leaf structure and evolution from a hydraulic perspective. Functional Plant Biology, Collingwood, v. 37, n. 6, p. 488-498, 2010.

DAMATTA, F. M.; RENA, A. B. Tolerância do café à seca. In: ZAMBOLIM, L. (Ed.). Tecnologias de produção de café com qualidade. Viçosa: UFV, 2001. p. 65-100.

DEMARTELAERE, A. C. F. et al. Utilização de polímero hidroabsorvente no meloeiro (Cucumis melon L.) sob diferentes lâminas de irrigação. Caatinga, Mossoró, v. 22, n. 3, p. 5-8, 2009.

DRANSKI, J. A. L. et al. Sobrevivência e crescimento do pinhão-manso em função do método de aplicação e formulações de hidrogel. Revista brasileira de Engenharia Agrícola e Ambiental, Campina Grande, v. 17, n. 5, p. 537542, 2013.

EL-HADY, O. A.; GHALY, N. F.; WANAS, S. E. Hydrophobic-hydrophilic combination for sandy soil conditioning and plantation: 1 . Growth response and water and fertilizers use efficiency by casuarina trees. AmericanEurasian Journal of Agricultural \& Environmental Science, Dubai, v. 4, n. 3, p. 332-342, 2008.

FERREIRA, D. F. Sisvar: a computer statistical analysis system. Ciência e Agrotecnologia, Lavras, v. 35, n. 6, p. 1039-1042, 2011.

HYDROPLAN-EB. Tecnologias de irrigação: ficha técnica e de segurança. 2001. Disponível em: <http://www. hydroplan-eb.com/i/ficha_tecnica_e_de_seguranca_HyA. pdf $>$. Acesso em: 24 jun. 2014.

JOVER, S. et al. Influence of rootstocks on photosynthesis in navel orange leaves: effects on growth, yield, and carbohydrate distribution. Crop Science, Madison, v. 52, n. 2, p. 836-848, 2012.

KAVANAGH, K. L.; PANGLE, R.; SCHOTZKO, A. D. Nocturnal transpiration causing disequilibrium between soil and stem predawn water potential in mixed conifer forests of Idaho. Tree Physiology, Oxford, v. 27, n. 4, p. 621-629, 2007.

LOPES, J. L. W. et al. Uso de hidrogel na sobrevivência de mudas de Eucalyptus urograndis produzidas com diferentes substratos e manejos hídricos. Ciência Florestal, Santa Maria, v. 20, n. 2, p. 217-224, 2010.

MACHADO, E. C. et al. Fluxo de seiva e fotossíntese em laranjeira 'Natal'com clorose variegada dos citros. Pesquisa Agropecuária Brasileira, Brasília, DF, v. 41, n. 6, p. 911-918, 2006.

MARQUES, P. A. A.; CRIPA, M. A. M.; MARTINEZ, E. H. Hidrogel como substituto da irrigação complementar em viveiro telado de mudas de cafeeiro. Ciência Rural, Santa Maria, v. 43, n. 1, p. 1-7, 2013.

MATTOS JÚNIOR, D. et al. Citros: manejo da fertilidade do solo para alta produtividade. Informações Agronômicas, Piracicaba, n. 128, p. 5-12, 2009.

OLIVEIRA, R. P.; SCIVITTARO, W. B.; NAKASU, B. H. Cultivares apirênicas de citros de mesa: nova alternativa para os produtores do Sul do Brasil. In: ENCONTRO NACIONAL DE FRUTICULTURA DE CLIMA TEMPERADO, 8., Fraiburgo, 2005. Palestras... Caçador: Embrapa, 2005. p. 13-21.

ORIKIRIZA, L. J. B. et al. Amending soils with hydrogels increases the biomass of nine tree species under non-water stress conditions. Clean Journal, Malden, v. 37, n. 8, p. 615-620, 2009. 
PEREZ-PEREZ, J. G. et al. Leaf water relations and net gas exchange responses of salinized Carrizo citrange seedlings during drought stress and recovery. Annals of Botany, Oxford, v. 100, n. 2, p. 335-345, 2007.

PIO, R. M. A qualidade e as exigências do mercado de tangerinas. Revista Brasileira de Fruticultura, Jaboticabal, v. 25, n. 3, p. 375-376, 2003.

PREVEDELlO, C. L.; LOYOLA, J. M. T. Efeito de polímeros hidroretentores na infiltração da água no solo. Scientia Agraria, Curitiba, v. 8, n. 3, p. 313-317, 2007.

R FOUNDATION FOR STATISTICAL COMPUTING (RFSC). $R$ : a language and environment for statistical computing. Vienna: RFSC, 2011.

REICHARDT, K. Capacidade de campo. Revista Brasileira de Ciência do Solo, Campinas, v. 12, n. 3, p. 211-216, 1988.

REWALD, B. et al. Phenotypic plasticity and water flux rates of citrus root orders under salinity. Journal of Experimental Botany, Oxford, v. 63, n. 7, p. 2717-2727, 2012.

RIBEIRO, R. V.; MACHADO, E. C. Some aspects of citrus ecophysiology in subtropical climates: re-visiting photosynthesis under natural conditions. Brazilian Journal of Plant Physiology, Rio Claro, v. 19, n. 4, p. 393-411, 2007.
RIBEIRO, R. V. et al. Seasonal and diurnal changes in photosynthetic limitation of young sweet orange trees. Environmental and Experimental Botany, Paris, v. 66, n. 2, p. 203-211, 2009.

SAAD, J. C. C.; LOPES, J. L. W.; SANTOS, T. A. Manejo hídrico em viveiro e uso de hidrogel na sobrevivência pós-plantio de Eucalyptus urograndis em dois solos diferentes. Engenharia Agricola, Jaboticabal, v. 29, n. 3, p. 404-411, 2009.

SOUZA, C. A. de et al. Crescimento em campo de espécies florestais em diferentes condições de adubações. Ciência Florestal, Santa Maria, v. 16, n. 3, p. 243-249, 2006.

TAIZ, L.; ZEIGER, E. Fisiologia vegetal. 4. ed. Porto Alegre: Artmed, 2009.

TOHIDI-MOGHADAM, H. R. et al. Response of six oilseed rape genotypes to water stress and hydrogel application. Pesquisa Agropecuária Tropical, Goiânia, v. 39, n. 3, p. 243-250, 2009.

VAle, G. F. R.; CARVAlho, S. P.; PAIVA, L. C. Avaliação da eficiência de polímeros hidroretentores no desenvolvimento do cafeeiro em pós-plantio. Coffee Science, Lavras, v. 1, n. 1, p. 7-13, 2006.

ZONTA, J. H. et al. Influência de diferentes turnos de rega e doses de hidroabsorvente no desenvolvimento inicial da cultura do café conillon (Coffea canephora pierre). Idesia, Arica, v. 27, n. 1, p. 29-34, 2009. 